\title{
A case of neonatal bronchomalacia, manifesting as severe poorly resolving bronchopneumonia
}

\author{
*Chandima Roshani Gunaratne ${ }^{1}$, Thusitha Sameera Kumarasiri² \\ Sri Lanka Journal of Child Health, 2020; 49(4): 409-411 \\ DOI: http://dx.doi.org/10.4038/sljch.v49i4.9280 \\ (Key words: Congenital bronchomalacia, pneumonia, neonates)
}

\section{Introduction}

Airway problems in neonates are often life threatening and raise challenging issues in diagnosis and management ${ }^{1,2}$. These could be classified as congenital or acquired lesions and congenital obstructive lesions can be intrinsic lesion in the airway, external compression or maldevelopment of the airway ${ }^{2}$. Congenital bronchomalacia is a form of maldeveloped airway resulting in collapse of floppy cartilaginous portion of the airway ${ }^{3}$ and is defined as "More than $50 \%$ dynamic collapse of the airways lumen, in the large right or left main-stem bronchi, and/or their respective divisions at the lobar or segmental bronchi, recorded at the end-expiratory point" 3 .

A high index of suspicion is needed for the diagnosis, especially in cases of recurrent wheeze/cough, breathlessness, easy fatiguability and episodic cyanosis, recurrent pneumonias owing to stagnation of secretions, life threatening events, repeated failed extubation attempts, persistent atelectasis or lobar hyperinflation on $\mathrm{CXR}^{1,3}$. We report a case of congenital bronchomalacia in a neonate presenting with poorly resolving bronchopneumonia complicated with type 1 respiratory failure.

\section{Case report}

A previously well one month old baby boy, who was a $3^{\text {rd }}$ born to non-consanguineous healthy parents with two healthy siblings, with an uneventful antenatal period, was transferred for further management. He was delivered at term (POA-37+5d), by elective caesarean section due to past two sections, with a birth weight of $3.735 \mathrm{~kg}$ and had an uneventful postnatal period. He was breastfed with no history of cough/choking while

\footnotetext{
${ }^{1}$ Senior Registrar in Neonatology, Sri Jayewardenepura General Hospital, Sri Lanka, ${ }^{2}$ Registrar in Emergency Medicine, National Hospital of Sri Lanka

*Correspondence: mail2roshii@yahoo.com

(iD)

orcid.org/ 0000-0002-0798-1986

(Received on 16 February 2020: Accepted after revision on 20 March 2020)

The authors declare that there are no conflicts of interest

Personal funding was used for the project.

Open Access Article published under the Creative Commons

Attribution CC-BY (C) (P)
}

feeding, no vomiting following feeds or contact history of tuberculosis or chronic cough. At $15^{\text {th }}$ day of life, he developed shortness of breathing, poor feeding and irritability with episodic cyanosis which lasted one week with no fever, needing admission to local hospital on day 21 .

He had severe respiratory distress with a low oxygen saturation $\left(\mathrm{SP}_{2}\right)$ of $82 \%$ in room air, needing intubation and mechanical ventilation for type 1 respiratory failure. He had coarse crepitations in bilateral lung fields with reduced air entry over right upper zone. However, he did not have features of evolving chronic lung disease, dysmorphism, heart murmur, organomegaly or neurological abnormality.

Investigations revealed neutrophil leucocytosis with high septic markers. Chest X-ray (CXR) showed bilateral extensive inflammatory shadows suggestive of severe bronchopneumonia with segmental compensatory hyperinflations. His renal and liver function tests and 2D echocardiogram were normal.

He was managed empirically with vancomycin, merapenem and oseltamivir along with frequent nebulisations. Intravenous (IV) fluids, along with methyl prednisolone and magnaesium sulphate infusions were commenced. Owing to persistent respiratory distress with poor response to treatment, he was extensively investigated with high resolution computer tomography scan-chest (HRCT) and fibrooptic laryngoscopy (FOL) which was normal with no evidence of laryngomalacia and HRCT showed "collapse consolidation in right upper lobe and bilateral lower lobes more in favour of infection with hyperinflated left upper lobe suspicious of bronchiolitis obliterans". Contrast enhanced CT chest (CECT) had excluded possible external compressive lesion in lungs and serial CXRs have shown persistent bilateral opacifications in lung fields (Figure 1). Immune screening was negative for HIV with normal white cells, immunoglobulin levels and functions. No fat globules in basic stool full report to suggest cystic fibrosis.

Specific antibiotics were commenced according to sensitivity patterns of blood and endo-tracheal aspirate cultures following microbiologist's opinion. Flexible bronchoscopy was performed by paediatric pulmonologist, showing "extensive functional collapse 
of bilateral segmental bronchi along with right main bronchus and a normal trachea". Broncho-alveolar lavage culture was negative for tuberculosis. The ultimate diagnosis of extensive congenital bronchomalacia was confirmed.

Baby was weaned off to humidified high flow nasal cannula (HHFNC) on $25^{\text {th }}$ day of ICU stay and was not able to be weaned off further due to frequent desaturations and persistent respiratory distress. Following multidisciplinary discussions, he was planned to manage conservatively. Following councelling of parents, baby was re-transferred with HHFNC for continuation of care.
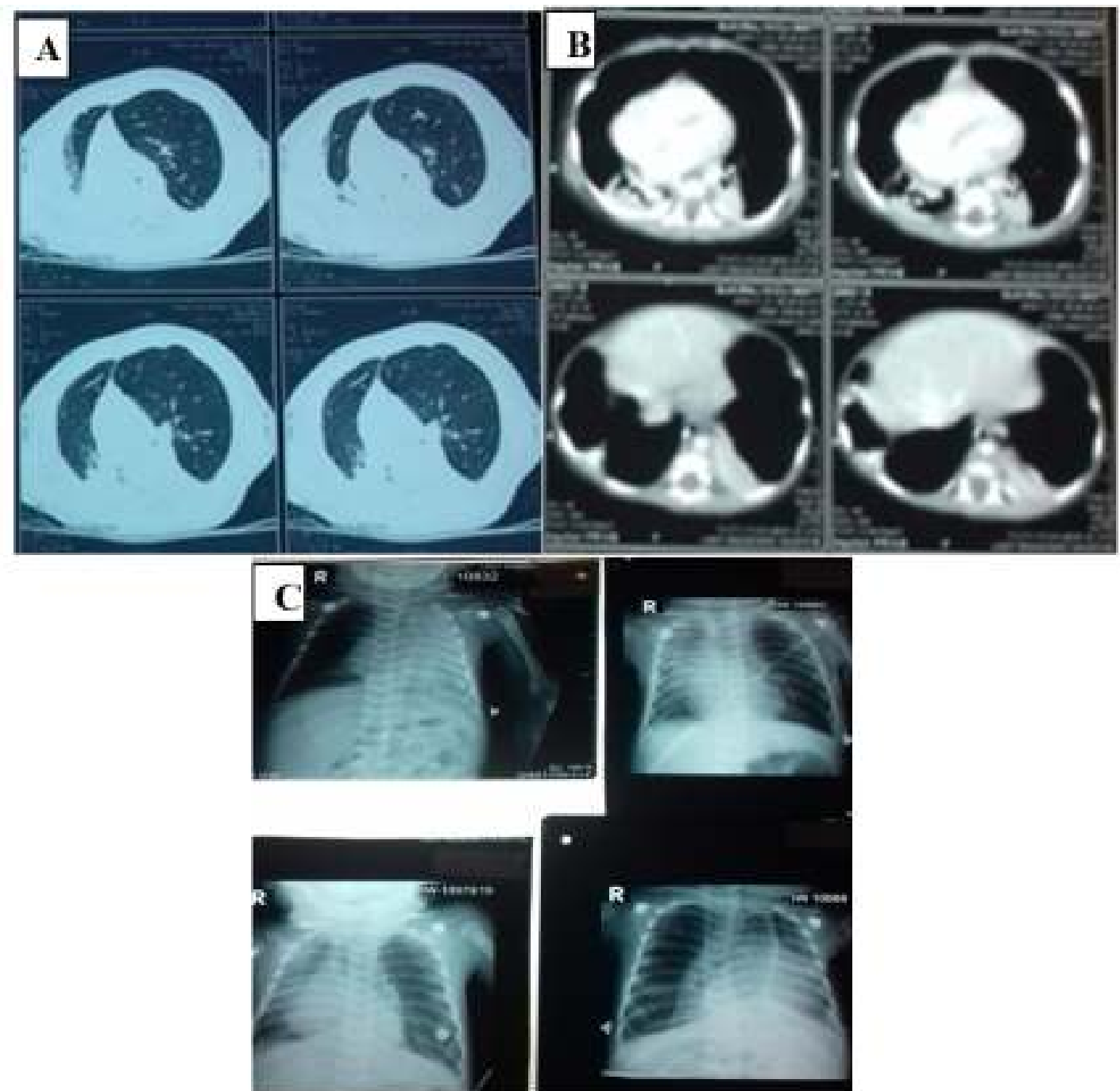

Figure 1: A-HRCT chest, B-CECT chest and $C$-Serial chest $x$-ray's showing multiple segmental lobar collapses with compensatory hyperinflations in lung fields HRCT: high resolution computer tomography, CECT: Contrast enhanced CT

\section{Discussion}

Congenital bronchomalacia usually occurs in 1-12 month old infants ${ }^{1,3}$ and the occurrence in neonates is rare $^{1}$. Hence, even though our patient presented with typical clinical symptoms of bronchomalcia with severe poorly resolving bronchopneumonia, the initial differential diagnoses were congenital structural lung problem or immune deficiency which were excluded later by investigations.

Many different investigations are available to diagnose and quantify the severity ${ }^{2}$. HRCT chest with 3D reconstruction angiogram may help demonstrate the airway and vascular anatomy providing useful data in planning surgery ${ }^{2,4}$. Moreover, bronchography, with and without positive end expiratory pressure (PEEP) provides dynamic information as CT underestimates presence of bronchomalacia in terms of extent and the severity and facilitate the determination of required PEEP to keep the airways open (Figure 2). However, both facilities are not freely available in our context $\mathrm{t}^{2,4}$. 


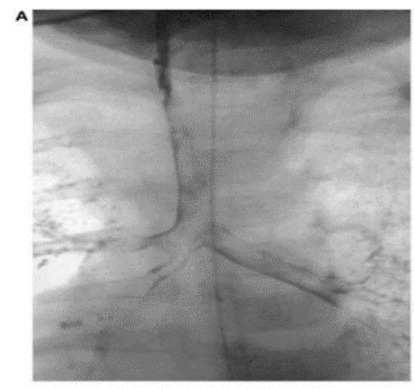

No PEEP

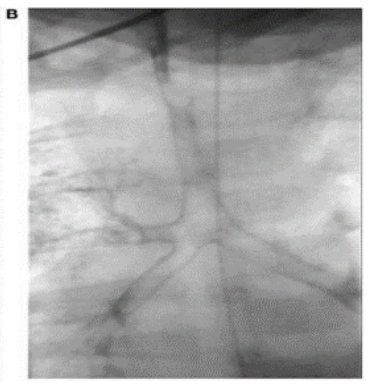

PEEP $10 \mathrm{cmH}_{2} \mathrm{O}$
Figure 02: Tracheo-bronchogram without (A) and with (B) PEEP; note the difference in calibre of the airway with PEEP

Bronchoscopy by flexible bronchoscopes or rigid ventilating bronchoscopes can pick up intrinsic airway lesions and collapse of the airway. Nevertheless, distal bronchomalacia may not be detected, thus an isotope ventilation scan, which is not freely avalable in our setting, can be of value in the diagnosis as well as determination of ventilator pressures required to ensure adequate ventilation of whole lung ${ }^{2,3,4}$ (Figure 3).

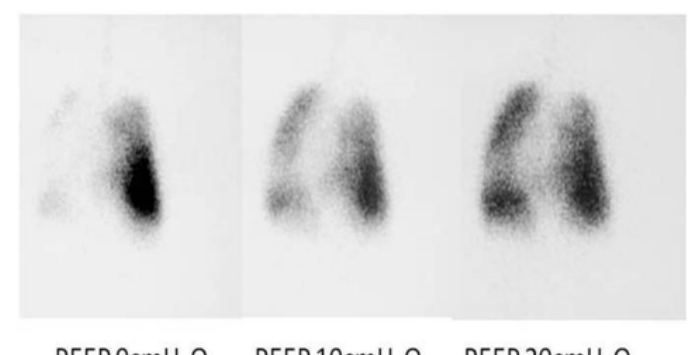

\section{PEEP $0 \mathrm{cmH}_{2} \mathrm{O}$ PEEP $10 \mathrm{cmH}_{2} \mathrm{O}$ PEEP $20 \mathrm{CmH}_{2} \mathrm{O}$}

Figure 3: Ventilation perfusion scan showing absence of lobar ventilation in the right lung, improving with increasing PEEP

Subsequent management of congenital bronchomalacia needs to be individualized and planned following precise assessment of the airway. Some may require ventilatory support in the form of continuous positive airway pressure (CPAP) to prevent lobar collapse ${ }^{1}$ while some may warrant operative interventions such as stenting or bronchopexy $y^{2,5}$. The type and need of intervention depends on the extent and severity of the airway involvement ${ }^{2,4}$. In our baby, due to extensive and severe involvement of segmental bronchi along with right main bronchus, surgical managment was suggested, however it was not performed due to the need of extensive stenting which carries higher post-op complication rates $^{2,4}$.
However, ongoing progress in this field with newer diagnostic tools and innovative management techniques, such as biodegradable stents and stem cellbased tracheo-bronchial transplants, will lead to a much better prognosis ${ }^{2,4,5}$ and will hopefully available in Sri Lanka in near future.

\section{References}

1. Denneny JC. Bronchomalacia in the neonate. Annals of Otology Rhinology and Laryngology 1985; 94(5 Pt 1): 466-9.

https://doi.org/10.1177/000348948509400510 PMid: 3901858

2. Quen Mok. Airway problems in neonates-A review of the current investigation and management strategies. Frontiers in Pediatrics 2017; 5: 60 .

https://doi.org/10.3389/fped.2017.00060

PMid: 28424763 PMCid: PMC5371593

3. Laberge JM, Puligandla P. Congenital malformations of the lungs and airways: Paediatric Respiratory Medicine. Second Edition. United States of America: Mosby; 2008: Chapter 64, Pages 907-41.

https://doi.org/10.1016/B978-032304048$8.50068-2$

4. Pillai JB, Smith J, Hasan A, Spencer D. Review of paediatric airway malacia and its management, with emphasis on stenting. European Journal of Cardio-Thoracic Surgery 2005; 27: 35-44.

https://doi.org/10.1016/j.ejcts.2004.10.001

PMid: 15621469

5. Mutabagani KH, Menke JA, McCoy KS, Besner GE. Bronchopexy for congenital bronchomalacia in the newborn. Journal of Pediatric Surgery 1999; 34(8): 1300-3. https://doi.org/10.1016/S0022-3468(99)90177$\mathrm{X}$ 